\title{
Computer based learning and comprehension of power generation cycles using a model programmed in Engineering Equation Solver (EES): Analysis of a Gas- Turbine Brayton cycle
}

\author{
C. Montagud*, J. Pons-Llinares*, M.C. Rodríguez-Monzonís** \\ * Instituto de Ingeniería Energética \\ Universitat Politècnica de València \\ València, Spain \\ ** Instituto de Ciencias de la Educación \\ Universitat Politècnica de València \\ València, Spain
}

\begin{abstract}
The comprehension of the influence of each design and operation parameter in a power generation cycle requires a complex analysis mainly due to coupled effects existing in these cycles and which is too hard to be assessed manually by the students during a practical session. Therefore, the use of a software tool specially developed for this purpose becomes essential. The present work presents a model of a Gas-Turbine Brayton cycle developed in EES (Engineering Equation Solver), which enables the student to make simulations in the software and easily determine the optimal design parameters that would maximize the electric energy production in this kind of cycle. In order to evaluate the usefulness of this tool, two questionnaires with the same questions were prepared and were provided to the students before and after the practical session. After analyzing the results of the questionnaires, and taking into account the comments provided by the students, it was concluded that using this methodology helps to stimulate the student's understanding during the session, and enables to verify that students put into practice their theoretical knowledge, being able to make a better transfer to more complicated and real applications in the future.
\end{abstract}

Keywords: Computer-aid learning and comprehension, engineering application, analysis and optimization of complex processes.

\section{Introduction}

\section{Context of the analyzed subject}

This paper presents a practical methodology to help the learning and comprehension of the students enrolled in a subject of the official master degree in "Energy Technology for a Sustainable Development" at the Universitat Politècnica de València (UPV) in Valencia, Spain. The master is divided in two main parts as shown in Figure 1: the first one comprises 4 compulsory fundamental subjects which cover 15 credits ECTS and the second, consists of 3 different specializations which cover the next 27 credits ECTS each. The subject that will be analyzed in this paper is called 'Introduction to Energy Technology', and it is one of the fundamental subjects taught during the first semester of the master.

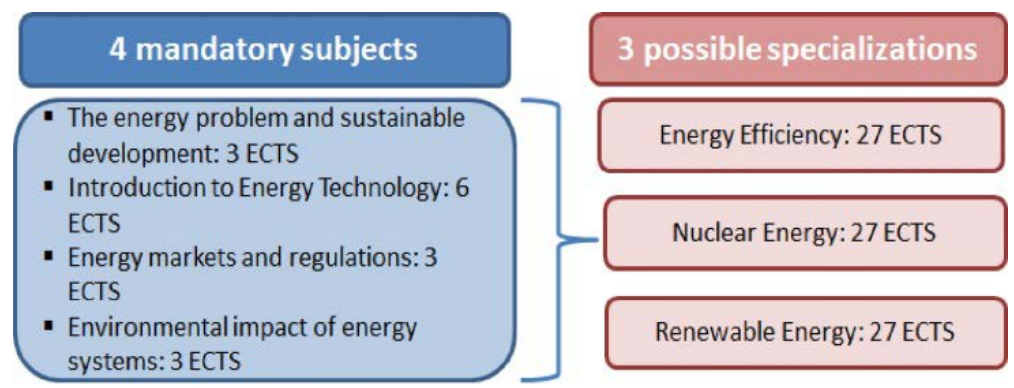

Figure 1. Structure of the "Master in Energy Technology for a Sustainable Development". 
"Introduction to Energy Technology" is divided into two main parts: the first one covers an overview of the different energy sources, where their main technological aspects are studied; and the second one, covers the study of power generation cycles where not only the components are analyzed but also the optimal design and operation parameters. Among the different practical lessons proposed, the one that is trying to be improved with the descriptive work here presented is dedicated to analyze the Gas- Turbine Brayton cycle for power generation.

The subject has been taught by the authors for four years now, i.e, since the 2009-2010 academic year. Since then, a systematic observation was carried out and the feedback information collected by the students was reflected on a diary according to Zabalza (2004). Further analysis of this information allowed to have evidence of the students' opinion and thus enabled the authors to improve in their teaching-learning process, especially during the practical lessons of the subject. It was observed that, it was too hard and time consuming to students to manually analyze the influence of each of the design and performance parameters in a power generation cycle in a single practical lesson which turned out to be very time consuming and unfeasible in the duration of the subject. Therefore, it was considered the need of providing students with a software tool able to easily identify the main performance and design parameters of this kind of cycles, study its influence in the cycle efficiency and learn to find their optimal values. This way, students could put into practice their theoretical knowledge by means of a software tool during the practical sessions of the subject.

This paper will present the results obtained for this teaching technique of the GasTurbine Brayton cycle which was recently carried out during the academic year 20132014. This same technique could be also applied to any other type of power generation cycle. The equations that describe the performance of the Gas- Turbine Brayton cycle were programmed in a software tool that was described and provided to the students who learned how to use it. The model developed is very friendly to use, and it helps students to develop some other similar models in their future work. Therefore, it also contributes to the development of some of the competences related to the master: decision making, system design, research skills, applying knowledge to the industrial practice, or performing energy audit.

The usefulness of this tool is assessed by means of a descriptive study on teaching strategy developed in class. To this end, two questionnaires with the same questions are provided to the students: one before and another one after the practical session. Then, the student can compare the answers provided before and after the practical session and determine whether it has been useful to improve their understanding during the practical session and to a latter extent in their learning process. Nevertheless, as there are many other factors that could influence the student's performance, the authors are aware that, through the strategy employed, it cannot be ensured that this is the only factor affecting the achieved progress in the student's learning and comprehension.

No empirical data are presented by the authors in this work for two main reasons: first of all, the process will be repeated during the next academic courses in order to improve the software itself; and second, more information will be compiled by the authors over the next several years of teaching in order to have a greater amount of data and be able to make a more complete diagnosis. 


\section{Gas- Turbine Brayton Cycle Model Developed}

Description of an ideal Gas- Turbine Brayton cycle

The Gas- Turbine Brayton cycle is a thermodynamic cycle that describes the operation of a constant pressure heat engine. Gas turbine engines use the Brayton Cycle. Although the Brayton cycle is usually run as an open system (and indeed must be run as such if internal combustion is used), it is conventionally assumed for the purposes of thermodynamic analysis that the exhaust gases are reused in the intake, enabling analysis as a closed system (Closed Brayton cycle). Figure 2 shows the components of a basic Gas- Turbine Brayton cycle: a gas compressor, a burner (or combustion chamber) and a gas turbine.
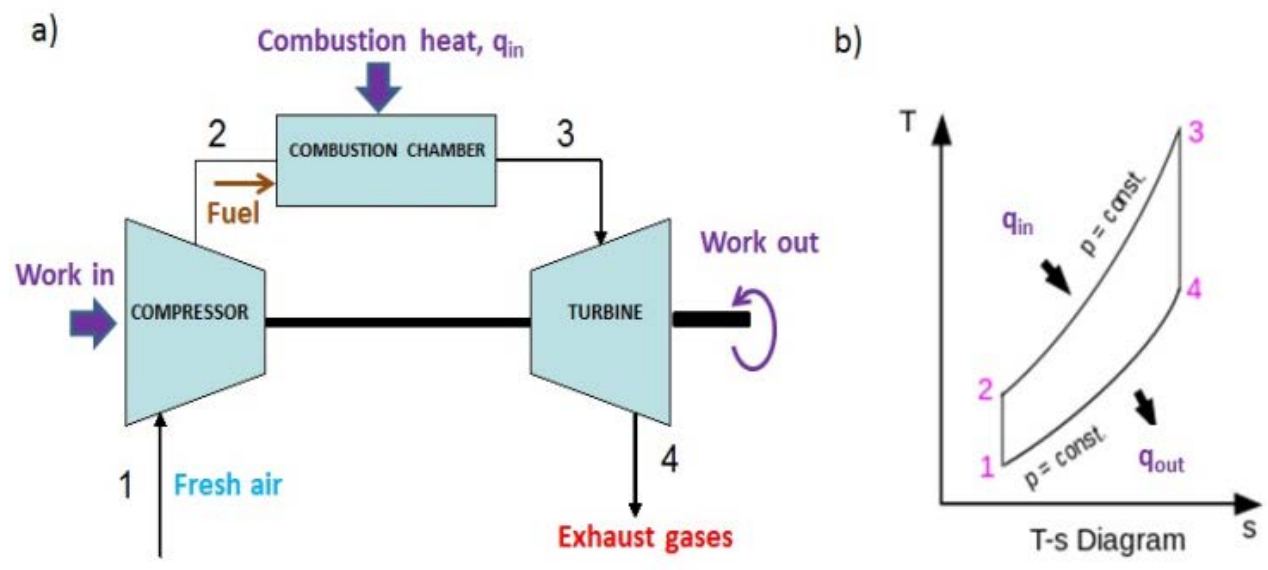

Figure 2. a) Gas- Turbine Brayton cycle: main components; b) T-s diagram of an ideal Gas- Turbine Brayton cycle.

There are four main processes that take place in a Gas- Turbine Brayton cycle:

1-2: Isentropic Compression. Ambient air is drawn into the compressor, where it is pressurized.

2-3: Isobaric Heat Transfer. The compressed air then is introduced in a combustion chamber, where fuel is burned, heating the compressed air at constant-pressure.

3-4: Isentropic Expansion. The heated and pressurized air enters the gas turbine (or series of turbines), and it expands obtaining useful energy. It should be stressed that some of the work extracted by the turbine is used to drive the compressor.

4-1: Isobaric Heat Rejection in the atmosphere. The exhaust gases in the gas turbine cool down at constant ambient pressure in contact with the atmosphere.

The efficiency of the power generation Gas- Turbine Brayton cycle is defined by equation (1):

$$
\eta_{\text {cycle }}=\frac{W_{\text {net, cycle }}}{W_{c}}
$$


Where $\mathrm{W}_{\text {net,cycle }}=\mathrm{W}_{t}-\mathrm{W}_{c}$, is the difference between the useful energy obtained in the turbine $\mathrm{W}_{t}$, and the consumed energy needed in the compressor, $\mathrm{W}_{c}$.

The calculation of heat and work per unit of mass of operating fluid can be expressed as the difference between the inlet and outlet enthalpy of the operating fluid at each component of the cycle.

\section{Inefficiencies of Real Gas- Turbine Brayton Cycles}

In real plants, each stage of the Gas- Turbine Brayton cycle is associated with irreversible processes, which reduce the overall efficiency. On one hand, turbine and compressor present irreversibilities that reduce the overall efficiency of the cycle. The isentropic efficiency of the turbine and the compressor is defined by equations (2) and (3) respectively, where subscript 'is' indicates isentropic values.

$$
\begin{aligned}
& \eta_{t}=\frac{W_{t}}{W_{t, i s}} \\
& \eta_{c}=\frac{W_{c, i s}}{W_{c}}
\end{aligned}
$$

Looking at the formulas (2) and (3), and taking into consideration that the efficiency takes values lower than $100 \%$, it can be concluded that the irreversibilities make the real work obtained in the turbine decrease, and the real compression work needed increase. The turbine efficiency directly reduces the work produced in the turbine and, therefore, the overall efficiency. The same thing happens with the compressor efficiency: the work needed in the compressor increases and reduces the overall efficiency. On the other hand, the heat transfer processes at the combustion chamber are not ideal and there exist associated irreversibilities. The heat combustion process will not be considered in the equations and the combustion chamber will be considered as a heat exchanger where the combustion heat is added to the compressed air at constant pressure.

\section{Increasing the Efficiency of Gas- Turbine Brayton Cycles: parameters of influence}

The optimization of the cycles for power generation highly depends on the right choice of the design parameters. There are four main design and/or operation parameters that have an influence in the overall cycle efficiency:

- $\quad$ Temperature of the air entering the turbine: the higher the inlet temperature at the turbine, the higher will be the expansion work obtained.

- Pressure ratio: relation between the inlet pressure at the turbine and the inlet pressure at the compressor (ambient pressure in open cycles). In principle, the greater the pressure ratio (greater inlet pressure at the turbine), the greater will be the efficiency of the cycle, but there is an optimum that will be analyzed by the students in the practical session.

- Isentropic efficiency of the turbine: the higher the isentropic efficiency, the higher the efficiency of the cycle.

- Isentropic efficiency of the compressor: the higher it is, the greater is the cycle efficiency. 


\section{Methods}

This section describes the steps and methodology that were followed during the teaching strategy followed in class.

The practical session took place in a computer lab where 22 students were grouped in pairs, carrying out a cooperative work as proposed by Slavin (1990), and Reynolds et al. (2013).

First, a brief review of the theoretical knowledge needed to develop the tasks corresponding to the practical session was carried out. The equations needed to characterize the Gas- Turbine Brayton cycle which had already been presented during previous theoretical lessons, were reminded to students in order to make sure that they had the background needed to follow the practical session. After this short review, the software tool was presented to the students, explaining them its main features and tools, and they were given some free time to get used to it.

Finally, a descriptive evaluation of the learning and comprehension of the students was carried out as presented in the following subsections.

\section{Software tool developed in the Engineering Equation solver}

In the Gas- Turbine Brayton cycle presented in this paper, there is a strong interdependency between the different design and operation parameters. Some of the states (2, 3, and 4), presented in Figure 2a, are interdependent. For each state, the thermodynamic properties need to be determined, and therefore, changing one design parameter implies that the student would need to redo all the calculations, which normally takes the student thirty minutes. As the duration of the practical session is two hours, it is unfeasible to make many parametric studies in order to find the optimal design and operation parameters for such a cycle. Therefore, there is a need to use a software tool in which the necessary equations to calculate each state are already programmed, so that the student can check the effect of each parameter in the cycle performance in a very fast and easy way. This kind of software tools become especially important when analyzing a real power generation plant, in which the thermodynamic cycle gets more complex as it includes several expansion and regeneration processes. With the aid of such software tools, it is possible to carry out several parametric studies and easily determine the optimal design parameters at any cycle independently of its complexity.

Engineering Equation Solver (EES: Engineering Equation Solver, 2015) was the software chosen to program the equations of the Gas- Turbine Brayton cycle. The EES is a general equation-solving program able to numerically solve a great number of coupled non-linear algebraic and differential equations. The main reason why it was chosen is the high accuracy of its thermodynamic and transport property database, available for hundreds of substances, which can be used with the equation solving capability. Among other features, the software allows carrying out parametric studies which are useful for optimization purposes.

Figure 3 presents the interface that was developed in EES and provided to the student in order to analyze the influence of each design parameter on the cycle performance.

Using this tool, the student can analyze the influence of each design parameter (which appear framed in Figure 3 on the cycle efficiency, just by changing its value and clicking on the 'Calculate' button For instance, they could determine the optimal 
pressure ratio by making a parametric study, as shown in Figure 4a. Figure 4b shows another parametric study carried out by the students where they could analyze the influence of the temperature inlet of the air in the turbine (T3 in Figure 3) in the efficiency of the cycle.
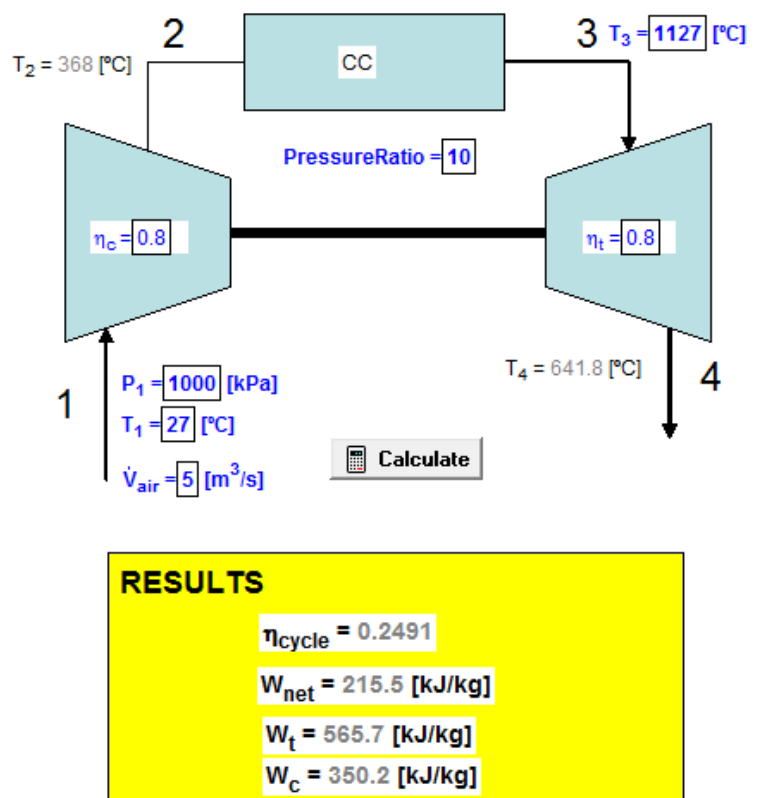

Figure 3. Basic Gas- Turbine Brayton cycle: diagram window programmed in EES.
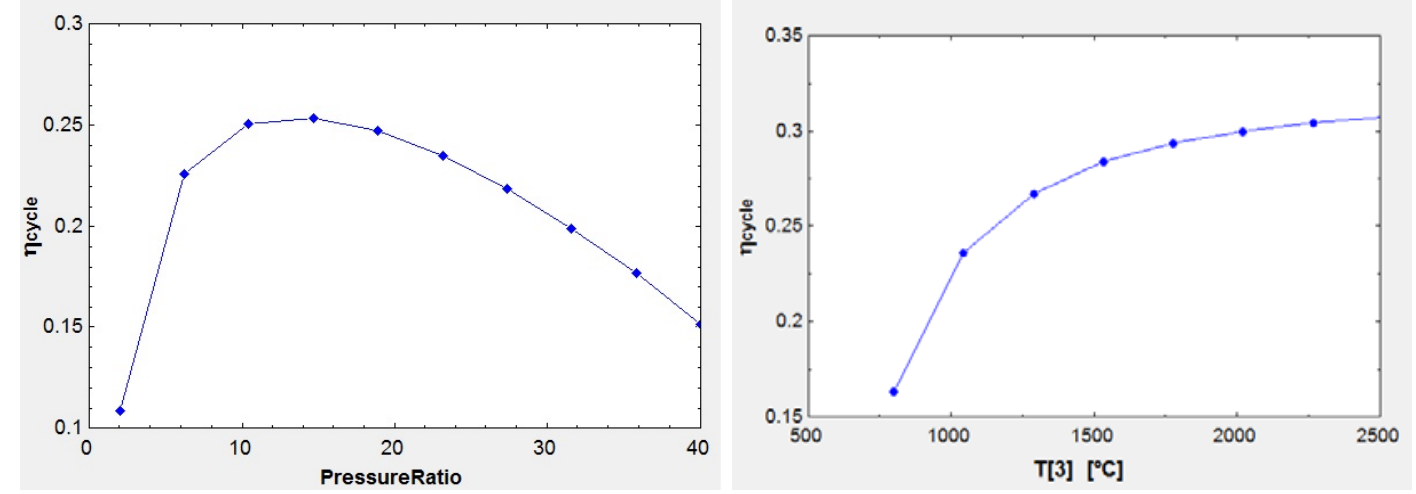

Figure 4. a) Influence of the pressure ratio in the cycle efficiency. b) Influence of the temperature of the air entering the turbine, T3, in the cycle efficiency.

\section{Evaluation of the learning and comprehension}

In order to carry out a diagnostic evaluation of prior knowledge as explained in Gibbs (2003), a simple questionnaire was provided to each group (2 students) before starting the practical session. The following questions were raised:

- Which are the three main components of a basic Gas- Turbine Brayton cycle and which process does it take place in each of them?

- Which are the four main design parameters that have an influence on the efficiency of the cycle? 
- How does the efficiency of the cycle vary when diminishing the value of each of the design parameters?

- Which is the main parameter that has a greater influence on the efficiency of the cycle?

Once the students had filled in the questionnaires, a brief theoretical review was carried out and the software tool was presented to the students so that they could program the equations of the cycle. Among the main features of the EES software, extra time was dedicated to the use of the parametric studies tool and the diagram window previously presented in Figure 3. After introducing the equations of the cycle in the EES software, students were asked to carry out several parametric studies (like the ones previously presented in Figure 4a and Figure $4 \mathrm{~b}$ in order to analyze the influence in the cycle efficiency of the following parameters: inlet pressure of the air at the turbine (P3), which varies modifying the value of the pressure ratio; inlet temperature of the air at the turbine, (T3); isentropic efficiency of the turbine, $\eta_{t}$, and isentropic efficiency of the compressor, $\eta_{c}$. Finally, the same questionnaire was provided to the students who were asked to answer exactly the same questions that were raised at the beginning of the practical session.

\section{Results and Discussion}

The results of the pre-test and post-test are presented in Figure 5a and Figure 5b respectively.
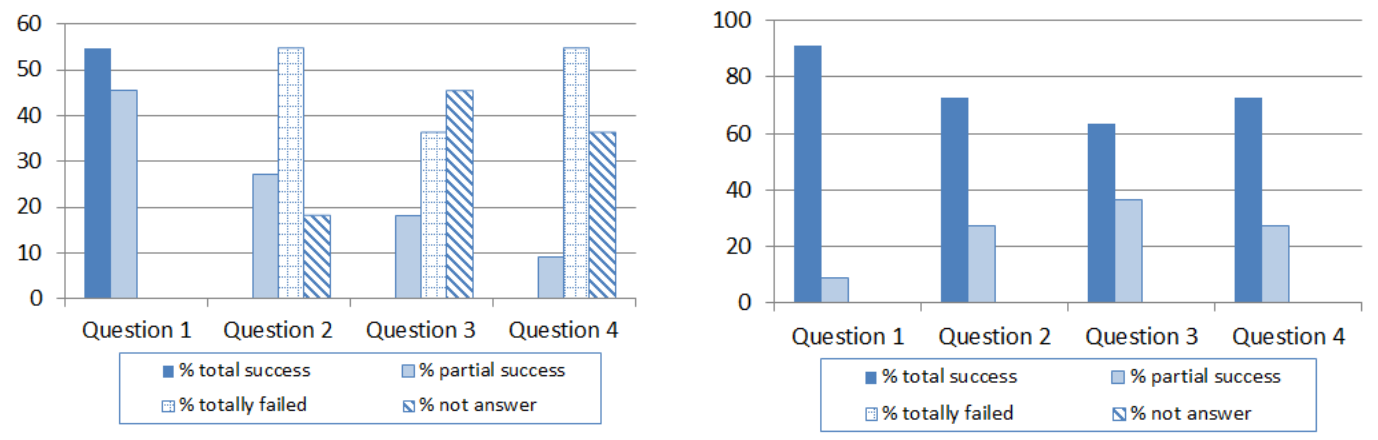

Figure 5. a) Results obtained in the pre-test. b) Results obtained in the post-test.

Results are expressed as the percentage of students that successfully answered to each question, those that partially succeeded in their answer, those that totally failed, and finally, those that left it blank notes. As shown in Figure 5a, most students knew the correct answer to the first question, since it was the most basic one, and they could easily answer it from the theoretical knowledge taught in other lessons which took place before the practical session. On the other hand, it can also be observed that, as the difficulty of the questions increased (question 2, 3 and 4), the percentage of students who totally succeeded dramatically decreased until values of $0 \%$ in all the questions, with a small percentage of $15 \%$ to $20 \%$ of students that partially answered correctly to questions from 2 to 4 . On the other hand, it can also be observed that approximately a fairly high percentage of students (in some cases higher than the 50\%) just left it blank notes, probably for fear of being judged by providing a wrong answer.

Figure 5b shows the results obtained after the practical session. As it can be observed, almost $100 \%$ of students correctly answered the first question. With respect to the second question, the percentage of students who answered correctly highly increased 
in comparison with the pre-test results, being the percentage of students that totally succeeded around $70 \%$. The same happened in questions 3 and 4 , in which the success rates clearly increased from $0 \%$ in the pre-test to $53 \%$ and $45 \%$ in the post -test respectively. It is observed that the percentage of students that totally failed the questions or those who leave it blank has become $0 \%$ in the pre -test up to values greater than $60 \%$ in the post-test, with maximum values of up to $70 \%$ on question 4 . Finally, taking a look at the results, it can be concluded that the practical session was useful for students who, after the practical session, had acquired a better comprehension of how the Gas- Turbine Brayton cycle performs as well as the influence of each of the design parameters on the efficiency of the cycle.

\section{Conclusions}

Based on the results observed in this descriptive study and considering the feedback provided by the students after the practical session, it was concluded that the developed tool and methodology followed helped to ease the student's comprehension during the session, making it possible to verify that it may have contributed to an improvement and stimulation in their personal learning process. Early feedback from students was very positive, as some of them informed the authors that they used it to prepare the exam, and confirmed their willing to use it in future works.

In order to allow a more complete analysis, the following future actions are proposed:

- Perform a qualitative analysis of the data through the development of surveys in which the students express their opinion on the usefulness of the methodology presented.

- Repeat the process along different academic courses, and take into account the feedback from students to improve both the software and the teaching strategy followed.

- $\quad$ Analyze if the software could be used in other subjects of the same master and get coordinated with the corresponding teachers in order to help them develop similar teaching strategies.

- Follow up with some students during their early working life and check whether they use it or they would find it useful at their work.

\section{References}

EES: Engineering Equation Solver. Retrieved January 21, 2015, from www.fchart.com/ees/

Gibbs, G. (2003) Uso estratégico de la evaluación en el aprendizaje. In Brown \& Glasner (Ed.). Evaluar en la universidad. Problemas y nuevos enfoques (pp. 6170). Madrid: Narcea.

Reynolds, W., Miller, G., \& Weiner, I. (Eds.) (2013). Cooperative Learning and Achievement: Theory and Research. Handbook of Psychology (2nd ed., Vol.7, pp. 199-212). Hoboken, NJ: Wiley.

Slavin, R.E. (1990). Cooperative learning. New Jersey: Prentice-Hall.

Zabalza, M. Á. (2004). Diarios de clase: un instrumento de investigación y desarrollo profesional, Madrid: Narcea. 